\title{
PROPRIEDADES TÉRMICAS DE GRÃOS DE TRIGO: DETERMINAÇÃO E MODELAGEM
}

\author{
Thermal properties of wheat grain: Determination and modelling
}

\author{
Deise Menezes Ribeiro' ${ }^{1}$, Paulo Cesar Corrêa ${ }^{2}$, Osvaldo Resende $^{3}$, Fernando Mendes Botelho $^{4}$
}

\begin{abstract}
RESUMO
Objetivou-se com o presente trabalho, avaliar as propriedades térmicas dos grãos de trigo, de uma variedade nacional (Aliança), durante o processo de secagem em estufa e ajustar modelos matemáticos que representem satisfatoriamente as variáveis estudadas em função do teor de água. Foram utilizados grãos de trigo com teor de água inicial de $26 \%$ b.s. secos até o teor final de 11 \%b.s. em estufa com ventilação forçada a $40^{\circ} \mathrm{C}$ e umidade relativa de $45 \%$. As variáveis estudadas foram o calor específico, obtido pelo método das misturas; a condutividade térmica, obtida por meio de um cilindro teoricamente infinito considerando a hipótese da condução unidimensional na direção radial; e a difusividade térmica, determinada indiretamente utilizando-se os valores experimentais da massa específica aparente, calor específico e condutividade térmica do trigo. Com base nos resultados obtidos, pode-se concluir que o calor específico e a condutividade térmica do trigo aumentam, enquanto a difusividade térmica apresenta redução dos seus valores com a elevação do teor de água e que estas propriedades térmicas do trigo variam linearmente com o teor de água, sendo satisfatoriamente representadas pelo modelo linear simples, para a faixa de umidade estudada.
\end{abstract}

Termos para indexação: Calor específico, condutividade térmica, difusividade térmica.

\begin{abstract}
The objective of this work was to evaluate the thermal properties of wheat grain, of a national variety, during the drying process in oven and to adjust mathematical models which represent satisfactorily these variables. Wheat grains with initial moisture content of $26 \%$ d.b. were dried in air forced oven up to $11 \%$ d.b. as the final moisture content. The specific heat was obtained by the method of the mixtures and the thermal conductivity by the utilization of a theoretically infinite cylinder considering the hypothesis of the conduction unidimensional in the radial direction. For thermal diffusivity was certain indirectly using itself the experimental values of the bulk density, specific heat and thermal conductivity of the wheat. Based on the results, it can be concluded that moisture content reduction promotes the specific heat and the thermal conductivity reduction but thermal diffusivity increase and which these thermal properties of the wheat vary lineally with water content, for the interval of studied humidity.
\end{abstract}

Index terms: Specific heat, thermal conductivity, thermal diffusivity.

(Recebido em 3 de junho de 2005 e aprovado em 17 de maio de 2006)

\section{INTRODUÇÃO}

O conhecimento das propriedades térmicas dos produtos vegetais é de suma importância para predizer e/ ou simular o comportamento de grãos e a quantidade de energia requerida quando submetidos a diferentes condições de secagem, aquecimento ou resfriamento.

Para Mohsenin (1980), grande parte das alterações internas de um produto que ocorrem durante as diversas etapas do processamento pode ser identificada conhecendo-se as suas principais propriedades térmicas: calor específico, condutividade e difusividade térmica. Estas características, próprias de cada produto, podem ser influenciadas pelo teor de água, temperatura e a massa específica aparente (CHANG, 1986).

O calor específico de um material pode ser definido como a quantidade de energia térmica (J) necessária para elevar a temperatura em um grau Celsius por unidade de massa (INCROPERA \& DEWITT, 1992). Diversos pesquisadores estudaram o comportamento dessa propriedade térmica com a variação do teor de água de vários materiais biológicos e observaram existir uma relação em que o aumento do teor de água provoca elevação dos valores de calor específico (ANDRADE et al., 2004; MOURA et al., 1998; SHARMA \& THOMPSON, 1973). Dentre os métodos para determinação do calor específico, o das misturas, proposto por Mohsenin (1980), é o mais comumente utilizado em pesquisas para determinação de calor específico de grãos e de sementes. Este método baseia-se em estabelecer o equilíbrio térmico de massas conhecidas de água e produto, com diferentes temperaturas, em calorímetro com capacidade calorífica conhecida. 
De acordo com Incropera \& Dewitt (1992), condutividade térmica define a quantidade de calor que é transmitida por unidade de tempo entre duas superfícies, através de uma unidade de área, devido a um gradiente de temperatura. Os problemas de transferência de calor em materiais biológicos durante a produção ou durante o manuseio e processamento podem incluir as interfaces sólido-líquido, sólido-gás, líquido-líquido e líquido-gás, como o aquecimento, resfriamento, congelamento, secagem ou desidratação de uma massa de sólidos ou de sólidos unitários. Em alguns casos, a transferência de calor é também acompanhada pela transferência de massa tal como ocorre nos processos de secagem e de desidratação (MOHSENIN, 1980).

Basicamente, são utilizados na determinação da condutividade térmica o método estacionário e o método transiente. Mohsenin (1980) e Reidy \& Rippen (1971), relataram que o método de medição de condutividade térmica em regime estacionário consiste da resolução da equação de condução de calor em estado estacionário, considerando uma amostra do produto modelada com uma forma geométrica simples (placas paralelas, cilíndricas ou esferas concêntricas). Este método, no entanto, não é recomendável para materiais biológicos, principalmente devido ao longo período de tempo necessário para atingir o regime estacionário, possibilitando modificações significativas no teor de água do produto. Estas dificuldades podem ser minimizadas pelo método de fluxo de calor transiente, com um período de teste consideravelmente reduzido. Um dos métodos mais usados, atualmente, para determinação da condutividade térmica de produtos agrícolas é baseado na transmissão de calor num cilindro infinito com uma fonte de calor linear central, como descrito por Andrade et al. (2004).

A condução de energia térmica em produtos agrícolas ocorre, normalmente, durante os processos de aquecimento ou resfriamento; no entanto, isto envolve o acúmulo ou dissipação de calor, que resultam em variações na distribuição de temperatura do material com o tempo. A velocidade de difusão do calor para dentro ou fora do material é dependente da difusividade térmica do produto (PABIS et al., 1988). Estudos realizados por Kazarian \& Hall (1965) confirmam que a magnitude da difusividade térmica influencia a cinética de secagem de grãos agrícolas.

A transferência de calor dentro de uma massa de grãos é um processo complexo. Devido às diferenças na composição química, teor de água e ar, é esperada uma variação das propriedades térmicas destes produtos (BOREM et al., 2002).
O efeito do teor de água sobre a condutividade e a difusividade térmica de diversos produtos vegetais tem sido objeto de estudo de vários autores, os quais verificaram relação diretamente proporcional entre as propriedades estudadas, podendo ser satisfatotiamente representadas por uma função linear (ANDRADE et al., 2004; KAZARIAN \& HALL, 1965; SUBRAMANIAN \& VISWANATHAN, 2003).

Diante do exposto, objetivou-se com o presente trabalho avaliar as propriedades térmicas dos grãos de trigo de uma variedade nacional, durante o processo de secagem em estufa e ajustar modelos matemáticos que as representem satisfatoriamente como função do teor de água.

\section{MATERIAL E MÉTODOS}

O presente trabalho foi realizado no Laboratório de Propriedades Físicas e Qualidade de Produtos Agrícolas pertencente ao CENTREINAR, localizado na Universidade Federal de Viçosa. Foram utilizados grãos de trigo, cultivar Aliança, colhidos com teor de água de, aproximadamente, 32 \%b.s. O produto foi armazenado em câmaras frias até o início dos testes.

Após o conhecimento do teor de água inicial do produto, efetuou-se a secagem em camada fina de aproximadamente $10 \mathrm{~kg}$ de grãos de trigo para obtenção dos demais níveis de teor de água, 26; 22; 20; 17; 14 e 11 $\%$ b.s., utilizando estufa com ventilação forçada à temperatura de $40{ }^{\circ} \mathrm{C}$ e umidade relativa de $45 \%$.

Após cada secagem, os teores de água do produto foram determinados pelo método padrão de estufa, $105 \pm 1{ }^{\circ} \mathrm{C}$, durante 24 horas, em três repetições (BRASIL, 1992).

Depois da secagem promoveu-se a homogeneização das amostras e a determinação das propriedades térmicas em três repetições.

$\mathrm{O}$ calor específico da massa de grãos $\left(\mathrm{C}_{\mathrm{p}}\right)$ foi obtido pelo método das misturas, segundo técnica descrita por Sasseron (1984). Neste método, o produto com massa e temperatura conhecidas foi colocado em um calorímetro, contendo água com temperatura e massa também conhecidas. Atingindo o equilíbrio térmico, o calor específico do produto foi determinado. Para a determinação da capacidade calorífica, utilizou-se a Equação 1:

$$
\mathrm{C}_{\mathrm{cal}}=\frac{\left[\mathrm{C}_{\mathrm{a}} \cdot \mathrm{m}_{2} \cdot\left(\mathrm{T}_{3}-\mathrm{T}_{2}\right)-\mathrm{C}_{\mathrm{a}} \cdot \mathrm{m}_{1} \cdot\left(\mathrm{T}_{1}-\mathrm{T}_{3}\right)\right]}{\left(\mathrm{T}_{1}-\mathrm{T}_{3}\right)}
$$

em que:

$\mathrm{C}_{\text {cal }}$ : capacidade calorífica do calorímetro $\left(\mathrm{kJ}^{\circ} \mathrm{C}^{-1}\right)$; 
$\mathrm{C}_{\mathrm{a}}$ : calor específico da água $\left(\mathrm{kJ} \mathrm{kg}^{-1}{ }^{\circ} \mathrm{C}^{-1}\right)$;

$\mathrm{m}_{1}$ : massa da água quente $(\mathrm{kg})$;

$\mathrm{m}_{2}$ : massa da água fria $(\mathrm{kg})$;

$\mathrm{T}_{1}$ : temperatura da água quente $\left({ }^{\circ} \mathrm{C}\right)$;

$\mathrm{T}_{2}$ : temperatura água fria $\left({ }^{\circ} \mathrm{C}\right)$;

$\mathrm{T}_{3}$ : temperatura de equilíbrio da água $\left({ }^{\circ} \mathrm{C}\right)$.

$\mathrm{O}$ calor específico dos grãos de trigo foi obtido por meio da seguinte expressão:

$$
\mathrm{C}_{\mathrm{p}}=\frac{\mathrm{C}_{\mathrm{a}} \cdot\left(\mathrm{m}_{1}+\mathrm{m}_{2}\right) \cdot\left(\mathrm{T}_{3}-\mathrm{T}_{5}\right)+\mathrm{C}_{\mathrm{cal}} \cdot\left(\mathrm{T}_{3}-\mathrm{T}_{5}\right)}{\mathrm{m}_{\mathrm{p}} \cdot\left(\mathrm{T}_{5}-\mathrm{T}_{4}\right)}
$$

em que:

$\mathrm{C}_{\mathrm{p}}$ : calor específico do produto $\left(\mathrm{kJ} \mathrm{kg}^{-1}{ }^{\circ} \mathrm{C}^{-1}\right)$;

$\mathrm{m}_{\mathrm{p}}$ : massa de produto $(\mathrm{kg})$;

$\mathrm{T}_{4:}^{\mathrm{p}}$ Temperatura do produto $\left({ }^{\circ} \mathrm{C}\right)$;

$\mathrm{T}_{5}$ : Temperatura de equilíbrio (água e produto) $\left({ }^{\circ} \mathrm{C}\right)$.

A condutividade térmica da massa granular foi determinada empregando-se um equipamento experimental baseado no método do cilindro teoricamente infinito, imerso em ambiente com temperatura controlada a $22{ }^{\circ} \mathrm{C}$, considerando a hipótese da condução unidimensional na direção radial, de acordo com metodologia descrita por Muir \& Chandra (1970). Para tal procedimento, utilizou-se um cilindro de alumínio com diâmetro e comprimento prédeterminados, contendo no centro um fio condutor de níquel-cromo, pelo qual se fez passar uma corrente de 1,14 A e tensão de 2,2 V. A temperatura foi monitorada por meio de termopares instalados na metade da altura do cilindro e distantes um centímetro entre si. A condutividade térmica da massa de trigo foi obtida em regime transiente, por coordenadas cilíndricas, conforme Equação 3:

$$
\mathrm{k}=\frac{\mathrm{Q}}{4 \cdot \pi \cdot\left(\mathrm{T}_{\mathrm{f}}-\mathrm{T}_{\mathrm{i}}\right)} \cdot \ln \left(\frac{\mathrm{t}_{2}-\mathrm{t}_{0}}{\mathrm{t}_{1}-\mathrm{t}_{0}}\right)
$$

em que:

$\mathrm{k}$ : condutividade térmica $\left(\mathrm{W} \mathrm{m}^{-1}{ }^{\circ} \mathrm{C}^{-1}\right)$;

$\mathrm{Q}$ : potência dissipada pela fonte por unidade de comprimento $\left(\mathrm{W} \mathrm{m}^{-1}\right)$;

$\mathrm{T}_{\mathrm{f}}$ : temperatura final do intervalo usado $\left({ }^{\circ} \mathrm{C}\right)$;

$\mathrm{T}_{\mathrm{i}}$ : temperatura inicial do intervalo usado $\left({ }^{\circ} \mathrm{C}\right)$;

$\mathrm{t}_{2}$ : instante final do intervalo usado para determinar a condutividade térmica (s)

$\mathrm{t}_{1}$ : instante inicial do intervalo usado para determinar a condutividade térmica $(\mathrm{s})$

$\mathrm{t}_{0}$ : fator de correção do tempo (s).
$\mathrm{O}$ fator de correção $\left(\mathrm{t}_{0}\right)$ tem a função de compensar a perda de parte do calor utilizado para aquecer o resistor elétrico no início do processo de transferência de energia para a massa de produto, calculado em função do logaritmo dos valores de tempo e das diferenças entre as temperaturas observadas ao longo do tempo e a temperatura inicial do sistema.

A difusividade térmica do produto foi determinada, indiretamente, utilizando-se os valores experimentais da massa específica aparente, do calor específico e da condutividade térmica, de acordo com a Equação 4:

$$
\alpha=\frac{\mathrm{k}}{\rho \cdot \mathrm{C}_{\mathrm{p}}}
$$

em que:

a: difusividade térmica $\left(\mathrm{m}^{2} \mathrm{~s}^{-1}\right)$;

$\mathrm{r}$ : massa específica $\left(\mathrm{kg} \mathrm{m}^{-3}\right)$.

A massa específica aparente das amostras foi determinada utilizando-se uma balança de peso hectolítrico, com capacidade para um quarto de litro, da marca Dallemole, em três repetições.

Os dados experimentais da condutividade térmica, do calor específico e da difusividade térmica foram submetidos à análise de regressão, utilizando-se as médias de três repetições para cada teor de água, sendo selecionado o modelo linear, em função da magnitude do coeficiente de determinação, para expressar a relação entre estas características e o teor de água do trigo.

\section{RESULTADOS E DISCUSSÃO}

Na Figura 1, estão apresentados os valores experimentais do calor específico da massa granular do trigo e estimados pelo modelo linear simples em função do teor de água.

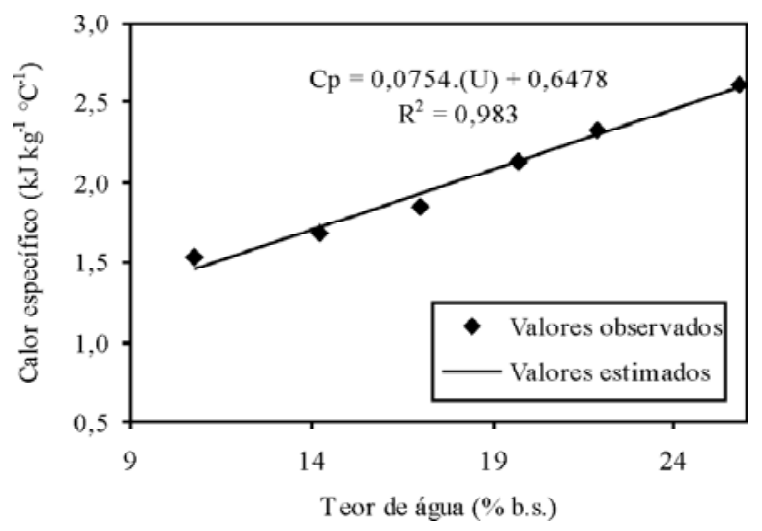

FIGURA 1 - Valores experimentais e estimados do calor específico dos grãos de trigo em função do teor de água (U). 
Observa-se que os valores do calor específico aumentaram com a elevação do teor de água, como acontece para a maioria dos produtos agrícolas, apresentando, para a faixa de umidade estudada, valores entre 1,52 a 2,60 $\left(\mathrm{kJ} \mathrm{kg}^{-1}{ }^{\circ} \mathrm{C}^{-1}\right)$. De acordo com Kazarian \& Hall (1965), a variação do calor específico do trigo em função do teor de água do produto, pode ser satisfatoriamente descrita pelo modelo linear simples, como observado no presente trabalho. Muir \& Viravanichai (1972) observaram que o calor específico do trigo, para a faixa de 30 a 42 \% b.s., não pode ser descrito pelo modelo linear simples, entretanto, para teores de água inferiores a $25 \%$ b.s. sua variação ocorre linearmente em função do teor de água.

Na Figura 2, estão apresentados os valores experimentais da condutividade térmica da massa granular do trigo e estimados pelo modelo linear simples em função do teor de água.

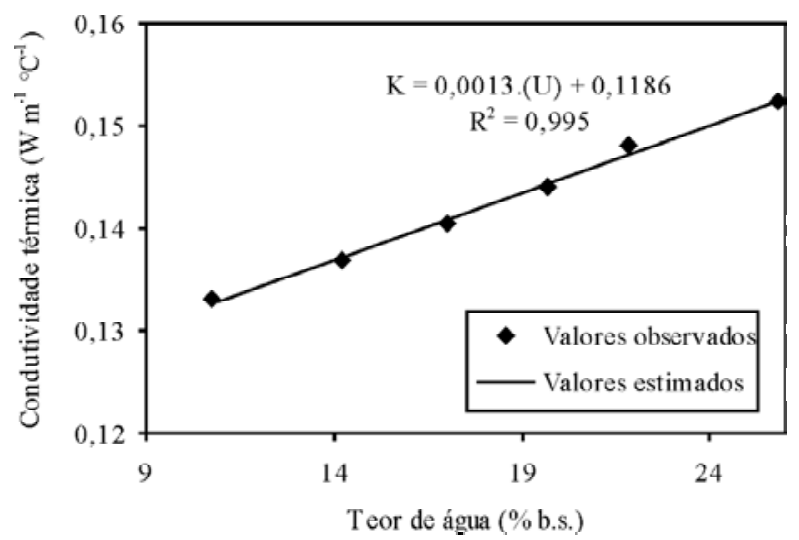

FIGURA 2 - Valores experimentais e estimados da condutividade térmica dos grãos de trigo em função do teor de água $(\mathrm{U})$.

Verifica-se que os valores da condutividade térmica também aumentaram linearmente com o teor de água do produto, conforme observado por Kazarian \& Hall (1965) para a faixa de umidade entre 5,71 e $25,63 \%$ b.s. Muir \& Chandra (1970) observaram que a condutividade térmica do trigo variou de 0,1382 a $0,1670\left(\mathrm{~W} \mathrm{~m}^{-1}{ }^{\circ} \mathrm{C}^{-1}\right)$ para teores de água entre 4 e $34 \%$ b.s. No presente trabalho, os valores de condutividade térmica da massa granular variaram de 0,1332 a $0,1523\left(\mathrm{~W} \mathrm{~m}^{-1}{ }^{\circ} \mathrm{C}^{-1}\right)$.

$\mathrm{Na}$ Tabela 1 estão apresentados os valores experimentais da massa específica aparente do trigo utilizados para o cálculo da difusividade térmica.
TABELA 1 - Valores experimentais da massa específica aparente do trigo em função do teor de água.

\begin{tabular}{cc}
\hline $\begin{array}{c}\text { Teor de água } \\
\text { (\%b.s.) }\end{array}$ & $\begin{array}{c}\text { Massa específica } \\
\text { aparente }\left(\mathbf{k g ~ m}^{-3}\right)\end{array}$ \\
\hline 25,79 & 672,26 \\
21,85 & 709,22 \\
19,67 & 730,47 \\
17,01 & 743,17 \\
14,22 & 750,98 \\
10,77 & 752,96 \\
\hline
\end{tabular}

Os valores experimentais da difusividade térmica da massa granular do trigo e estimados pelo modelo linear simples são apresentados na Figura 3. Verifica-se que os valores da difusividade térmica do trigo reduziram-se linearmente com o aumento do teor de água do produto, variando de $11,58 \times 10^{-8}$ a $8,69 \times 10^{-8}$ $\left(\mathrm{m}^{2} \mathrm{~s}^{-1}\right)$. Na literatura, observa-se uma intensa variabilidade nos valores de difusividade térmica para a massa de trigo de diferentes variedades, em função do teor de água, conforme pode ser observado na Tabela 2. Sabe-se que vários fatores influenciam a quantidade de calor que atravessa uma massa granular, desta forma, os valores da difusividade térmica podem variar entre produtos e variedades devido, principalmente, à sua composição, massa específica, porosidade e teor de água.

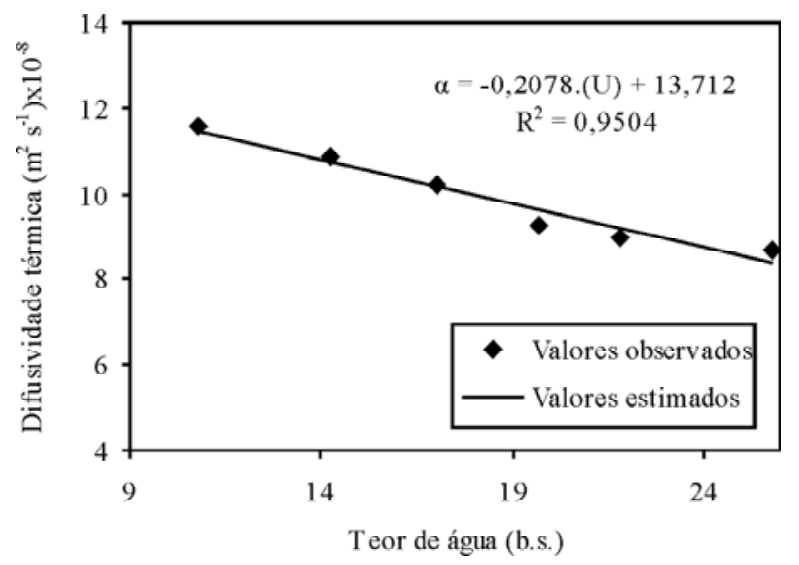

FIGURA 3 - Valores experimentais e estimados da difusividade térmica da massa granular de trigo $(\alpha)$ em função do teor de água (U). 
TABELA 2 - Valores de difusividade térmica do trigo encontrados na literatura para diferentes teores de água.

\begin{tabular}{ccc}
\hline Teor de água $(\%$ b.s. $)$ & Difusividade térmica $\left(\mathbf{1 0}^{-\mathbf{8}} \mathbf{~ m}^{\mathbf{2}} \mathbf{~ s}^{\mathbf{- 1}}\right)$. & Referências \\
\hline 10,77 a 25,79 & 8,54 a 7,52 & ASAE $(2001)$ \\
10 & 11,5 & ASAE (2001) \\
0,68 a 25 & 9,03 a 7,99 & Kasarian \& Hall (1965) \\
10 a 25 & 11,58 a 8,69 & Presente trabalho \\
\hline
\end{tabular}

\section{CONCLUSÕES}

Os resultados obtidos permitiram concluir que:

O calor específico e a condutividade térmica do trigo aumentam com a elevação do teor de água, para a cultivar e faixa de umidade estudada.

Os valores de difusividade térmica reduzem com o aumento do teor de água.

As propriedades térmicas analisadas variam linearmente em função do teor de água, sendo satisfatoriamente representadas, para a faixa de umidade estudada, pelo modelo linear simples.

\section{REFERÊNCIAS BIBLIOGRÁFICAS}

ANDRADE, E. T.; COUTO, A. M.; QUEIROZ, D. M.; PEIXOTO, A. B. Determinação de propriedades térmicas de grãos de milho. Ciência e Agrotecnologia, Lavras, v. 28, n. 3, p. 488-498, 2004.

ASAE. Thermal properties of grain and grain products: ASAE standard D243.3. Saint Joseph, 2001.

BOREM, F. M.; RIBEIRO, R. C. M. S.; CORRÊA, P. C.; PEREIRA, R. G. F. A. Propriedades térmicas de cinco variedades de café cereja descascado. Revista Brasileira de Engenharia Agrícola e Ambiental, Campina Grande, v. 6, n. 3, p. 475-480, 2002.

BRASIL. Ministério da Agricultura e Reforma Agrária. Regra para análise de sementes. Brasília, DF, 1992. 365 p.

CHANG, C. S. Thermal conductivity of wheat, corn, and grain sorghum as affected by bulk density and moisture content. Transactions of the ASAE, Saint Joseph, v. 29, n. 5, p. 1447-450, 1986.
INCROPERA, F. P.; DEWITT, D. P. Fundamentos de transferência de calor e de massa. 3. ed. Rio de Janeiro: Guanabara Koogan, 1992. 380 p.

KAZARIAN, E. A.; HALL, C. W. Thermal properties of grains. Transactions of the ASAE, Saint Joseph, v. 8, n. 1, p. 33-38, 1965.

MOHSENIN, N. N. Thermal properties of foods and agricultural materials. New York: Gordon and Breach Science, 1980. 407 p.

MOURA, S. C. S. R.; GERMER, S. P. M.; JARDIM, D. C. P.; SADAHIRA, M. S. Thermophysical properties of tropical fruit juices. Brazilian Journal of Food Technology, Campinas, v. 1, n. 1/2, p. 70-76, 1998.

MUIR, W. E.; CHANDRA, S. Thermal conductivity of spring wheat at low temperatures. Transactions of the ASAE, Saint Joseph, n. 70, p. 805, 1970.

MUIR, W. E.; VIRAVANICHAI, S. Specific heat of wheat. Journal Agricultural Engineering Research, New York, v. 17, p. 338-342, 1972.

PABIS, S.; JAYAS, D. S.; CENKOWSKI, S. Grain drying. New York: J. Wiley \& Sons, 1988. 303 p.

REIDY, G. A.; RIPPEN, A. L. Methods for determining thermal conductivity in foods. Transactions of the ASAE, Saint Joseph, v. 14, n. 2, p. 248-254, 1971.

SASSERON, J. L. Avaliação de propriedades físicas e curvas de secagem, em camadas finas, de amêndoas de cacau (Theobroma cacao L.). 1984. 61 f. Dissertação (Mestrado em Engenharia Agrícola) - Universidade Federal de Viçosa, Viçosa, 1984. 
SHARMA, D. K.; THOMPSON, T. L. Specific heat and thermal conductivity of sorghum. Transactions of the ASAE, Saint Joseph, v. 16, n. 1, p. 114-117, 1973.
SUBRAMANIAN, S.; VISWANATHAN, R. Thermal properties of minor millet grains and flours. Biosystems Engineering, [S.1.], v. 84, n. 3, p. 289-296, 2003. 